\title{
Déficit associé à l'alcool : recettes publiques et coûts pour la société associés à l'alcool au Canada
}

\author{
Adam Sherk, Ph. D.
}

Diffuser cet article sur Twitter

\section{Résumé}

Cet aperçu offre une comparaison entre les recettes publiques provenant de la vente et de la distribution d'alcool et les coûts pour la société associés à la consommation d'alcool, et ce, pour l'année 2014. Les données de Statistique Canada font état de recettes publiques de 10,9 milliards de dollars. Toutefois, ce montant est contrebalancé par des coûts nets pour la société de 14,6 milliards de dollars, comme le rapporte le projet Coûts et méfaits de l'usage de substances au Canada, un projet de surveillance nationale de la consommation de substances. Les coûts pour la société sont constitués des soins de santé, de la perte de productivité économique, de la justice pénale et de divers autres coûts directs. Bien que les recettes de vente d'alcool soient considérées comme un avantage pour les coffres de l'État, une comptabilisation tenant compte des coûts engagés montre que les provinces et les territoires du Canada subissent plutôt un déficit associé à l'alcool, pour un total de 3,7 milliards de dollars à l'échelle nationale.

Mots-clés : consommation d'alcool, coûts, coûts pour la société, projet Coûts et méfaits de l'usage de substances au Canada, déficit associé à l'alcool

\section{Introduction}

Les recettes publiques du Canada provenant de la vente et de la distribution d'alcool sont considérées comme profitables pour l'État, dans la mesure où ces recettes sont susceptibles d'être ensuite redirigées vers les soins de santé et l'éducation. En effet, il est évident, à l'examen des comptes publics, que la vente d'alcool est lucrative, rapportant des recettes considérables au gouvernement fédéral et aux gouvernements provinciaux $^{1}$. Toutefois, comme plus de $75 \%$ des Canadiens adultes ont consommé de l'alcool au cours de l'année précédente ${ }^{2}$, ces recettes sont contrebalancées par des coûts, considérables et croissants ${ }^{3}$, associés à l'exposition à grande échelle de la population à l'alcool. Ces coûts pour la société sont constitués des soins de santé, e la perte de productivité, de la justice pénale et de divers autres coûts directs.

Statistique Canada consigne, depuis un certain temps, les recettes publiques provenant de la vente de boissons alcoolisées sous la forme de droits d'accise fédéraux, de bénéfice net des régies des alcools provinciales et de taxe de vente (taxe de vente harmonisée [TVH], taxe de vente provinciale [TVP] et taxe sur les produits et services [TPS] $)^{1}$. Toutefois, il s'est écoulé plus de dix ans avant que le Canada ne se dote, en 2018, d'un système national de surveillance de la consommation de substances, soit le projet Coûts et méfaits de l'usage de substances au Canada (CEMUSC) ${ }^{3}$. Ce projet fournit une comptabilisation détaillée et exhaustive des coûts pour la société associés à huit substances psychoactives, dont l'alcool. L'alcool est la substance qui a coûté le plus cher au Canada en 2014, à savoir 14,6 milliards de dollars, soit plus que le tabac (12,0 milliards de dollars) et beaucoup plus que les opioïdes $(3,5$ milliards de dollars) et le cannabis (2,8 milliards de dollars) ${ }^{3}$.

Cet aperçu répond à la question : en tenant compte des recettes nettes et des

\section{Points saillants}

- Le Canada subit un déficit associé à l'alcool d'environ 3,7 milliards de dollars par année si on comptabilise à la fois les recettes publiques et les coûts pour la société provenant de sources établies.

- Les recettes publiques étaient de 10,9 milliards de dollars en 2014, mais ce montant a été plus que contrebalancé par des coûts pour la société de 14,6 milliards de dollars, comme le rapporte le projet Coûts et méfaits de l'usage de substances au Canada.

- Les coûts pour la société sont constitués des soins de santé, de la perte de productivité, de la justice pénale et de divers autres coûts directs.

- Au sein des provinces, et sur la base d'un calcul par habitant, c'est en Alberta que les recettes publiques sont les plus faibles et que le déficit associé à l'alcool a été le plus élevé.

coûts nets pour la société, les gouvernements du Canada ont-ils un surplus ou un déficit associé à l'alcool?

\section{Méthodologie}

Le cadre conceptuel utilisé par les sources de données décrites dans cet article est un scénario hypothétique, dans lequel l'exposition de la population à la consommation d'alcool est nulle et l'a toujours été. Aucune source, pas plus que cet aperçu, ne défend l'idée que la consommation d'alcool devrait être nulle : il s'agit simplement d'un moyen de comptabiliser les 
recettes publiques et les coûts pour la société causés par l'alcool ou qui lui sont attribués.

Les données provinciales et territoriales sur les recettes nettes des régies des alcools et des recettes publiques provenant de la vente d'alcool sont tirées de la base de données du CANSIM de Statistique Canada pour l'exercice 2014-2015². Les données téléchargées sont plus détaillées que celles présentées dans cet article, où j’ai utilisé le titre de tableau « bénéfice net des régies des alcools » et le titre de ligne " droits d'accise et droits à l'importation fédéraux " mais j'ai regroupé toutes les autres taxes et catégories de recettes sous la rubrique " taxe de vente et autres recettes ».

Le projet CEMUSC détaille les coûts économiques engagés par la société en fonction de 19 catégories et de 4 domaines (soins de santé, perte de productivité économique, justice pénale et autres coûts directs). Les données provinciales et territoriales de 2014 dans ces quatre catégories de coûts ont été consultées au moyen de l'outil interactif de visualisation du projet (https://www.cemusc.ca/consulter-les -donnees/) le 4 décembre 2019. La méthodologie utilisée pour chaque domaine et celle du projet CEMUSC sont décrites ailleurs $^{3,4}$. Le projet CEMUSC sous-estime les coûts des soins de santé dans la province de Québec, car des données manquent dans certaines catégories pour cette province.

Pour chaque province et territoire, ainsi que pour l'ensemble du Canada, les surplus ou déficits totaux nets ont été calculés en faisant la différence entre les recettes nettes et les coûts nets. J'utilise les termes "recettes nettes " et " coûts nets » puisque les coûts (lorsqu'on parle des recettes nettes) et les économies (lorsqu'on parle des coûts nets) ont déjà été comptabilisés. Par exemple, dans la catégorie de recettes nettes de "bénéfice net des régies des alcools » les coûts incorporables, les dépenses administratives et les salaires des employés ont déjà été déduits des ventes brutes. Pour ce qui est des coûts des soins de santé, une faible quantité d'alcool pouvant avoir un léger effet de protection sur certaines affections, comme le diabète ${ }^{5}$ ou la cardiopathie ischémique chez les femmes ${ }^{6}$, les économies pour le système de soins de santé qui y sont associées ont déjà été incluses dans les chiffres de " coût net ». Les chiffres par habitant ont été calculés à partir des effectifs de la population au $1^{\text {er }}$ juillet 2014 ?

\section{Résultats}

D'importantes recettes publiques - soit un total de près de 10,9 milliards de dollars provenaient de la vente d'alcool en 2014 (tableau 1). Toutefois, ce montant a été largement contrebalancé par des dépenses de 14,6 milliards de dollars en raison de la consommation d'alcool, ce qui a donné lieu à un déficit national d'environ 3,7 milliards de dollars en 2014.

Parmi les catégories de recettes, le bénéfice net des régies des alcools est le plus important à l'échelle nationale, soit 5,7 milliards de dollars (52\%), suivi de la taxe de vente et d'autres revenus pour 3,7 milliards de dollars (34\%) et des droits d'accise fédéraux pour 1,5 milliard de dollars $(14 \%)$. Ce bénéfice net des régies des alcools s'est révélé l'élément le plus

TABLEAU 1

Recettes nettes, coûts nets et déficit net des administrations associés à l'alcool, par administration et au Canada, 2014

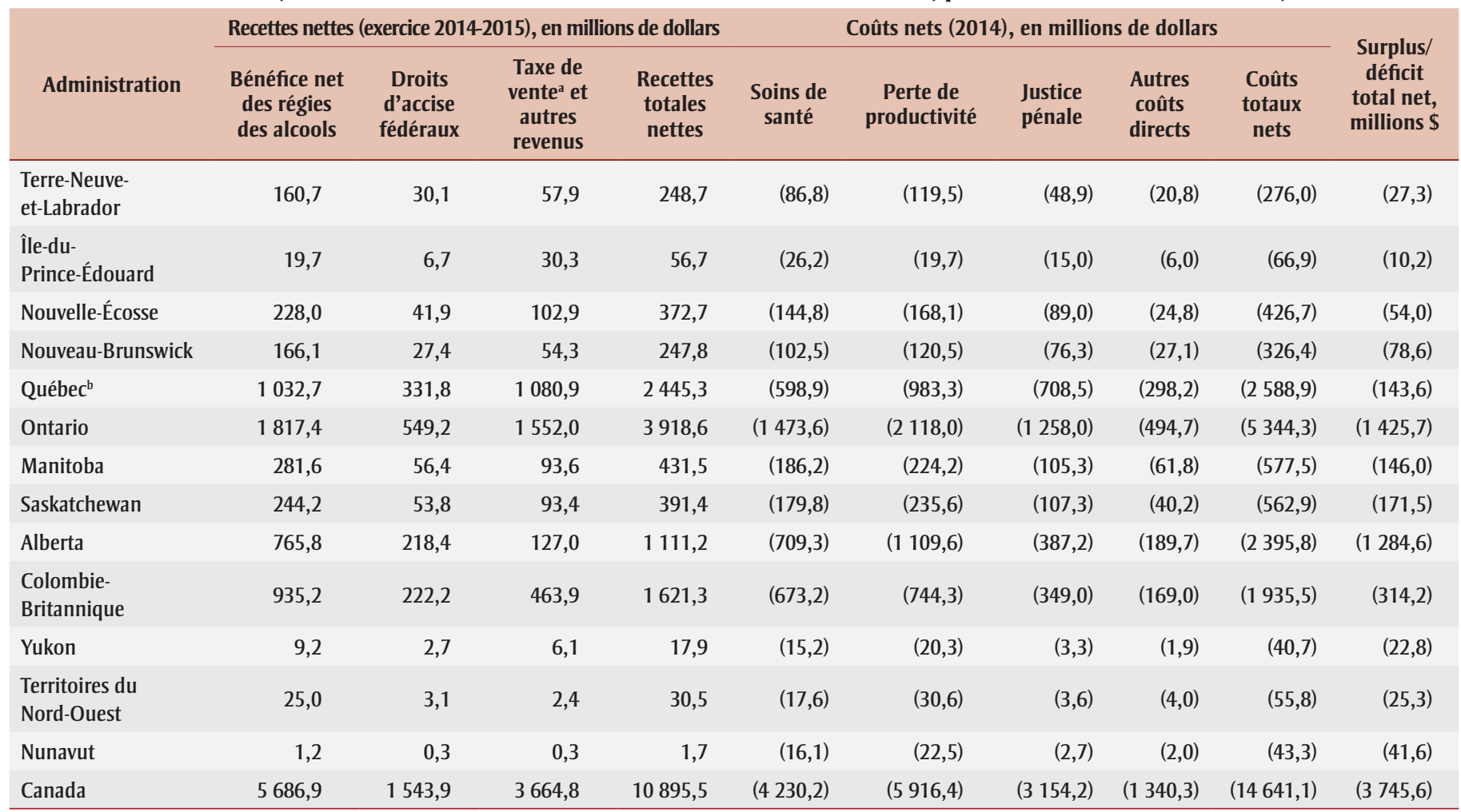

Remarque : Les nombres entre parenthèses sont négatifs.

a Taxe de vente provinciale (TVP), taxe de vente harmonisée (TVH) ou taxe sur les produits et services (TPS).

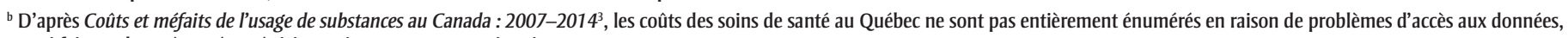
ce qui fait que les coûts présentés ici constituent une sous-estimation. 
important des recettes publiques dans toutes les administrations sauf à l'île-duPrince-Édouard et au Québec, où la taxe de vente et les autres revenus forment la catégorie la plus importante.

L'analyse des coûts engagés montre que la perte de productivité économique constituait la proportion la plus importante des coûts associés à l'alcool, soit 5,9 milliards de dollars (40\%), suivie des coûts du système de soins de santé pour 4,2 milliards de dollars (29\%), des dépenses en justice pénale pour 3,2 milliards de dollars (22\%) et des autres coûts directs pour 1,34 milliard de dollars (9\%). Il est à noter que le projet CEMUSC sous-estime les coûts des soins de santé au Québec, car des données manquent dans plusieurs catégories de soins de santé ${ }^{3}$.

L'analyse des recettes publiques ainsi que des coûts et des déficits par habitant révèle des différences régionales considérables (tableau 2). De toutes les provinces, c'est en Alberta que les recettes par habitant sont les plus faibles, soit 272 \$ par personne (pp), et que les coûts par habitant sont les plus élevés, soit 587 \$ pp, donnant ainsi lieu à un déficit par personne (315 \$) représentant plus du double de celui de la province classée au deuxième rang ainsi que près de six fois la moyenne nationale. Le Québec présente le déficit par habitant le plus faible, sachant toutefois que ce ne sont pas tous les coûts de soins de santé qui ont été comptabilisés. Terre-Neuve-et-Labrador (52 \$ pp), la Nouvelle-Écosse (58 \$ pp), l'île-du-PrinceÉdouard (70 \$ pp), le Nouveau-Brunswick (104 \$ pp) et l’Ontario (105 \$ pp) présentent des déficits par habitant en deçà de la moyenne nationale (106 \$ pp). Les déficits dans les territoires sont considérablement plus élevés que cette moyenne nationale.

\section{Analyse}

Une comptabilisation des recettes publiques et des coûts pour la société associés à la vente et à la consommation d'alcool au Canada montre que la conviction d'un surplus associé à l'alcool est erronée. Dans les 13 administrations, les coûts pour la société sont plus élevés que les recettes publiques, ce qui conduit à un " déficit associé à l'alcool " global de 3,7 milliards de dollars en Canada en 2014.

Cette synthèse comporte des limites dans la mesure où les sources de données utilisées pour estimer les recettes du gouvernement et les coûts pour la société offrent une comptabilisation incomplète et sont susceptibles d'être sous-estimées. Les

TABLEAU 2

Recettes nettes, coût net et déficit net par habitant, par administration et au Canada, 2014

\begin{tabular}{lrrr} 
& \multicolumn{3}{c}{ Par personne, en dollars } \\
\cline { 2 - 4 } \multicolumn{1}{c}{ Administration } & $\begin{array}{c}\text { Recettes publiques } \\
\text { nettes }\end{array}$ & Coût net & Surplus ou déficit net \\
\hline Terre-Neuve-et-Labrador & 471,0 & $(522,6)$ & $(51,6)$ \\
Île-du-Prince-Édouard & 393,3 & $(463,7)$ & $(70,4)$ \\
Nouvelle-Écosse & 397,2 & $(454,6)$ & $(57,5)$ \\
Nouveau-Brunswick & 326,5 & $(430,1)$ & $(103,6)$ \\
Québec $^{\text {a }}$ & 300,0 & $(317,6)$ & $(17,6)$ \\
Ontario & 287,8 & $(392,5)$ & $(104,7)$ \\
Manitoba & 337,4 & $(451,5)$ & $(114,1)$ \\
Saskatchewan & 351,6 & $(505,8)$ & $(154,1)$ \\
Alberta & 272,1 & $(586,7)$ & $(314,6)$ \\
Colombie-Britannique & 344,4 & $(411,2)$ & $(66,7)$ \\
Yukon & 483,0 & $(1095,9)$ & $(613,0)$ \\
Territoires du Nord-Ouest & 695,3 & $(1271,5)$ & $(576,2)$ \\
Nunavut & 48,5 & $(1203,7)$ & $(1155,2)$ \\
Canada & 307,5 & $(413,2)$ & $(105,7)$ \\
\hline
\end{tabular}

Remarque : Les nombres entre parenthèses sont négatifs.

a D'après Coûts et méfaits de l'usagé de substances au Canada : 2007-2014², les coûts des soins de santé au Québec ne sont pas entièrement énumérés en raison de problèmes d'accès aux données, ce qui fait que les coûts présentés ici constituent une sous-estimation. entreprises de fabrication d'alcool et les employés des commerces d'alcool paient des impôts sur leurs revenus de société ou de particulier au gouvernement fédéral ou aux gouvernements provinciaux. Cet élément devrait faire partie des recettes indirectes du gouvernement associées à la consommation d'alcool au Canada. Par ailleurs, comme je l'ai souligné plus haut, le cadre conceptuel de cette étude est un scénario hypothétique dans lequel la population de référence n'est nullement exposée à l'alcool, or cette étude ne recommande pas d'utiliser ce scénario autrement que dans le cadre d'une comptabilisation fondée sur ce scénario. Dans ce scénario hypothétique de référence, les entrepreneurs et les sociétés feraient partie d'autres secteurs de l'économie que celui de l'industrie de l'alcool, or il est difficile de déterminer l'effet économique global de cette diversion de l'énergie et du capital de la production et de la vente d'alcool vers d'autres secteurs.

Les coûts pour la société associés à la consommation d'alcool saisis dans le cadre du projet CEMUSC sont susceptibles d'être considérablement sous-estimés, étant donné que certaines catégories de coûts n'ont pas pu être énumérées en raison de contraintes de méthodologie ou de données. Le projet ne tient pas compte, par exemple, du coût de la douleur et de la souffrance humaines vécues par les personnes et par leur entourage social et associées à des affections de santé causées par l'alcool. Il ne tient pas non plus compt de la perte de productivité économique résultant de l'incarcération, des coûts tout au long de la vie de l'ensemble des troubles causés par l'alcoolisation fœtale et les coûts des traitements privés ${ }^{3}$.

\section{Conclusion}

Les coûts pour la société, à savoir les soins de santé, la perte de productivité économique, la justice pénale et d'autres coûts directs, étaient considérablement plus élevés que les recettes gouvernementales associées à l'alcool dans l'ensemble des provinces et des territoires en 2014. À l'échelle nationale, les recettes publiques de 10,9 milliards de dollars sont en deçà des coûts pour la société, qui sont de 14,6 milliards de dollars d'après l'étude CEMSUC, donnant donc lieu à un déficit associé à l'alcool 3,7 milliards de dollars. Il est évident que l'on prend à Pierre pour donner à Paul. 


\section{Remerciements}

Ce travail a été rendu possible grâce à une bourse postdoctorale des Instituts de recherche en santé du Canada.

\section{Conflits d'intérêts}

AS était un rédacteur invité de ce numéro de la Revue PSPMC, mais il s'est soustrait à toutes les décisions éditoriales concernant ce manuscrit.

\section{Contributions de l'auteur et avis}

AS est responsable de l'ensemble de l'article.

Le contenu de l'article et les points de vue qui y sont exprimés n'engagent que les auteurs et ne correspondent pas nécessairement à ceux du gouvernement du Canada.

\section{Références}

1. Statistique Canada. Bénéfice net des régies des alcools et recettes des administrations publiques de la vente des boissons alcoolisées (x 1000). Fréquence : annuelle. Tableau : 10-100012-01 (anciennement CANSIM 1830025). Géographie : Canada, province ou territoire [Internet]. Ottawa (Ont.) : Statistique Canada; [consultation le 4 déc. 2019]. En ligne à : https:// www150.statcan.gc.ca/t1/tbl1/en/tv .action?pid $=1010001201$

2. Sherk A, Gilmore W, Churchill S, Lensvelt E, Stockwell T, Chikritzhs T. implications of cardioprotective assumptions for national drinking guidelines and alcohol harm monitoring systems. Int J Environ Res Public Health. 2019;16(24). doi:10.3390/ijerph16244 956.

3. Groupe de travail scientifique sur les coûts et les méfaits de l'usage de substances au Canada. Coûts et méfaits de l'usage de substances au Canada (2007-2014). (Preparé par le Canadian Institute for Substance Use Research et le Centre canadien sur les dépendances et l'usage de substances). Ottawa (Ont.) : Centre canadien sur les dépendances et l'usage de substances; 2018.
4. Sorge JT, Young M, Maloney-Hall B, et al. Estimation of the impacts of substance use on workplace productivity: a hybrid human capital and prevalence-based approach applied to Canada. Can J Public Health. 2020; 111(2):202-11. doi:10.17269/s41997-019 $-00271-8$.

5. Knott C, Bell S, Britton A. Alcohol consumption and the risk of type 2 diabetes: a systematic review and dose-response meta-analysis of more than 1.9 million individuals from 38 observational studies. Diabetes Care. 2015;38(9):1804-12. doi:10.2337/dc15 -0710 .

6. Roerecke M, Rehm J. The cardioprotective association of average alcohol consumption and ischaemic heart disease: a systematic review and meta-analysis. Addiction. 2012;107(7): 1246-60. doi:10.1111/j.1360-0443.2012 $.03780 . \mathrm{x}$.

7. Statistique Canada. Estimation de la population au 1er juillet, par âge et par sexe. Fréquence : annelle. Tableau : 17-10-0005-01 (anciennement CANSIM 051-0001). Géographie : Canada, province ou territoire [Internet]. Ottawa (Ont) : Statistique Canada; [consultation le 4 déc. 2019]. En ligne à : https://www150.statcan.gc.ca/t1/tbl1 $/$ fr/tv.action?pid $=1710000501$ \&request _locale $=\mathrm{fr}$ 